Received: 28 July 2017

Accepted: 13 December 2017

Published online: 08 January 2018

\section{Increased pulse wave transit time after percutaneous coronary intervention procedure in CAD patients}

\begin{abstract}
Lizhen $\mathrm{Ji}^{1,2}$, Chengyu Liu ${ }^{1}{ }^{1}$, Peng Li ${ }^{1}$, Xinpei Wang ${ }^{1}$, Changchun Liu ${ }^{1}$ \& Yinglong Hou ${ }^{3}$
Pulse wave transit time (PWTT) has been widely used as an index in assessing arterial stiffness. Percutaneous coronary intervention $(\mathrm{PCI})$ is usually applied to the treatment of coronary artery disease (CAD). Research on the changes in PWTT caused by PCl is helpful for understanding the impact of the $\mathrm{PCl}$ procedure. In addition, effects of stent sites and access sites on the changes in PWTT have not been explored. Consequently, this study aimed to provide this information. The results showed that PWTT significantly increased after $\mathrm{PCI}(p<0.01)$ while the standard deviation (SD) of PWTT time series had no statistically significant changes $(p=0.60)$ between before and after PCI. Significantly increased PWTT was found in the radial access group $(p<0.01)$, while there were no significant changes in the femoral access group $(p>0.4)$. Additionally, PWTT in the left anterior descending (LAD) group significantly increased after $\mathrm{PCI}(p<0.01)$, but the increase that was found in the right coronary artery (RCA) group was not significant $(p>0.1)$. Our study indicates that arterial elasticity and left ventricular functions can benefit from a successful PCI procedure, and the increase of peripheral PWTT after PCI can help to better understand the effectiveness of the procedure.
\end{abstract}

Pulse wave transit time (PWTT) refers to the time interval that a pulse wave propagates through the distance between two arterial sites ${ }^{1,2}$. It is the time interval from the electrocardiogram (ECG) R-wave peak to the foot of the synchronized photoplethysmography (PPG) signal ${ }^{3}$. Regardless of the resting or exercise state $e^{4,5}$, an increase in PWTT generally indicates a decrease in arterial stiffness, which is often associated with cardiovascular events ${ }^{6,7}$. As one of the most common cardiovascular diseases, coronary artery disease (CAD) often requires treatment by percutaneous coronary intervention (PCI), and the demand for PCI has increased tremendously. Bundhun et al. have found that there are no significant differences in adverse outcomes between same day discharge and overnight hospital stay ${ }^{8}$. Therefore, a better explanation of the changes in PWTT of CAD patients, especially between before and after PCI, is helpful to understand the effectiveness of PCI. Significantly lower carotid-femoral aortic PWV after PCI has been reported ${ }^{9}$, but there is insufficient literature discussing peripheral PWTT. In this study, we explore the changes of peripheral PWTT within $24 \mathrm{~h}$ after a PCI procedure.

PCI can be carried out through radial access or femoral access; the safety and effectiveness of these procedures have been assessed in numerous studies ${ }^{10-14}$. In 2012, Bertrand et al. compiled a meta-analysis of studies regarding PCI performed via radial access or femoral access. The results suggest that the procedure performed through radial access has better outcomes with respect to complications, bleeding, and death ${ }^{13}$. Hannan et al. ${ }^{14}$ also found a trend of mortality reduction for patients treated through radial access, though the difference was not significant. Consequently, this study aims to explore the influence of the different artery access points on the changes in PWTT between before and after the PCI procedure.

The left anterior descending (LAD) artery and the right coronary artery (RCA) are two main sites for performing $\mathrm{PCI}^{15}$. Previous studies found good outcomes in patients with revascularization in isolated $\mathrm{LAD}^{16,17}$. It is known that the LAD is approximately twice as large as the RCA ${ }^{18,19}$. Here, we investigate if stents in the isolated LAD or RCA can influence changes in peripheral PWTT and whether there will be different effects for these cases.

${ }^{1}$ School of Control Science and Engineering, Shandong University, Jinan, 250061, China. ${ }^{2}$ Institute of Environment and Ecology, College of Geography and Environment, Shandong Normal University, Jinan, 250014, China. ${ }^{3}$ Department of Cardiology, Shandong Provincial Qianfoshan Hospital, Shandong University, Jinan, 250014, China. Correspondence and requests for materials should be addressed to C.L. (email: changchunliu@sdu.edu.cn) 


\begin{tabular}{|c|c|c|c|c|c|c|}
\hline \multirow[b]{2}{*}{ Subject No. } & \multicolumn{2}{|c|}{ PWTT (ms) } & \multicolumn{2}{|c|}{$\begin{array}{l}\text { SD of PWTT } \\
(\mathrm{ms})\end{array}$} & \multicolumn{2}{|c|}{ SBP (mmHg) } \\
\hline & $\begin{array}{l}\text { Before } \\
\text { PCI }\end{array}$ & \begin{tabular}{|l} 
After \\
PCI
\end{tabular} & $\begin{array}{l}\text { Before } \\
\text { PCI }\end{array}$ & \begin{tabular}{|l} 
After \\
PCI
\end{tabular} & $\begin{array}{l}\text { Before } \\
\text { PCI }\end{array}$ & $\begin{array}{l}\text { After } \\
\text { PCI }\end{array}$ \\
\hline 1 & 194 & 206 & 3.81 & 3.61 & 150 & 150 \\
\hline 2 & 215 & 226 & 3.04 & 3.76 & 137 & 131 \\
\hline 3 & 202 & 193 & 6.89 & 3.72 & 117 & 118 \\
\hline 4 & 185 & 201 & 4.41 & 6.22 & 140 & 138 \\
\hline 5 & 200 & 205 & 4.18 & 3.91 & 130 & 130 \\
\hline 6 & 182 & 196 & 3.34 & 2.86 & 117 & 117 \\
\hline 7 & 220 & 243 & 7.31 & 6.93 & 140 & 143 \\
\hline 8 & 197 & 222 & 4.00 & 4.13 & 134 & 126 \\
\hline 9 & 201 & 219 & 2.33 & 3.73 & 124 & 126 \\
\hline 10 & 169 & 178 & 1.96 & 2.52 & 115 & 110 \\
\hline 11 & 192 & 208 & 2.59 & 5.18 & 130 & 139 \\
\hline 12 & 190 & 200 & 1.62 & 3.21 & 121 & 126 \\
\hline 13 & 207 & 203 & 2.64 & 1.67 & 125 & 129 \\
\hline 14 & 217 & 209 & 6.68 & 8.76 & 125 & 117 \\
\hline 15 & 195 & 189 & 2.91 & 2.06 & 136 & 132 \\
\hline 16 & 217 & 217 & 5.83 & 7.28 & 130 & 135 \\
\hline 17 & 216 & 207 & 7.21 & 3.09 & 136 & 131 \\
\hline 18 & 207 & 211 & 2.98 & 2.69 & 119 & 115 \\
\hline 19 & 219 & 229 & 4.51 & 3.99 & 114 & 107 \\
\hline 20 & 252 & 269 & 7.66 & 6.72 & 121 & 117 \\
\hline 21 & 209 & 205 & 3.72 & 4.46 & 135 & 138 \\
\hline 22 & 180 & 177 & 3.51 & 3.43 & 143 & 148 \\
\hline 23 & 198 & 201 & 2.86 & 2.82 & 130 & 130 \\
\hline 24 & 196 & 208 & 4.46 & 5.32 & 129 & 130 \\
\hline 25 & 201 & 195 & 1.57 & 2.26 & 133 & 126 \\
\hline 26 & 204 & 216 & 2.43 & 2.32 & 117 & 115 \\
\hline 27 & 230 & 234 & 5.97 & 5.89 & 127 & 108 \\
\hline Mean & 204 & 210 & 4.09 & 4.17 & 129 & 127 \\
\hline SE & 3 & 4 & 0.35 & 0.35 & 2 & 2 \\
\hline$p$ & \multicolumn{2}{|l|}{$<0.01$} & \multicolumn{2}{|l|}{0.60} & \multicolumn{2}{|l|}{0.18} \\
\hline
\end{tabular}

Table 1. Results of PWTT, SD of PWTT and SBP from before and after PCI for all patients.

\section{Results}

PWTT differences between before and after PCI. Table 1 shows the results of PWTT and standard deviation (SD) of PWTT time series, along with SBP from before and after PCI for each patient. Statistical results showed that PWTT significantly increased after PCI $(210 \pm 4 \mathrm{~ms}$ vs. before $204 \pm 3 \mathrm{~ms}, p<0.01)$ while the SD of PWTT and SBP revealed no significant difference (before $4.09 \pm 0.35 \mathrm{~ms}$ vs. after $4.17 \pm 0.35 \mathrm{~ms}, p=0.60$; before $127 \pm 2 \mathrm{mmHg}$ vs. after $125 \pm 3 \mathrm{mmHg}, p=0.18$ ).

Effects of access sites and stent sites on PWTT. The changes in PWTT (value after PCI minus value before PCI) are shown in Fig. 1 with specific annotation for the radial and femoral access sites (A) and for the LAD and RCA stent sites (B). As shown in Fig. 1(A), the majority of patients (85\%) underwent PCI through radial access, and among these, sixteen patients exhibited increased PWTT, six had decreased PWTT, and one had no change after the PCI procedure. Four patients (15\%) underwent the procedure through femoral access when the radial access failed, and among these patients, two had increased PWTT while the others had decreased PWTT after the procedure. The mean changed value was $7.9 \mathrm{~ms}$ for the radial access group, and $-2.5 \mathrm{~ms}$ for the femoral access group. Eighteen patients accepted stent implantation in the LAD, which was approximately $67 \%$ of the total patients, and the other nine patients (approximately $33 \%$ ) accepted stent implantation in the RCA, as shown in Fig. 1(B). In the LAD group, twelve patients exhibited an increased PWTT after PCI, while six patients had a decrease. The mean value of PWTT change was $7.2 \mathrm{~ms}$. In the RCA group, six patients exhibited an increase, one remained unchanged and the other two had a decrease in the PWTT, resulting in a mean change of $4.7 \mathrm{~ms}$.

Figure 2(A) shows the results of PWTT in both groups divided by access site, before and after the PCI procedure. PWTT in the radial access group after PCI significantly increased ( $210 \pm 4 \mathrm{~ms}$ vs. before $202 \pm 4 \mathrm{~ms}$, $p<0.01)$, while a slight decrease was found in the femoral access group ( $211 \pm 9 \mathrm{~ms}$ vs. before $214 \pm 6 \mathrm{~ms}$, $p>0.4)$, though there was no significant difference between before and after PCI.

PWTT before and after PCI are shown for both the LAD and RCA groups in Fig. 2(B). The results show that PWTT in the LAD group after PCI significantly increased $(210 \pm 5 \mathrm{~ms}$ vs. before $202 \pm 5 \mathrm{~ms}, p<0.01)$. While 


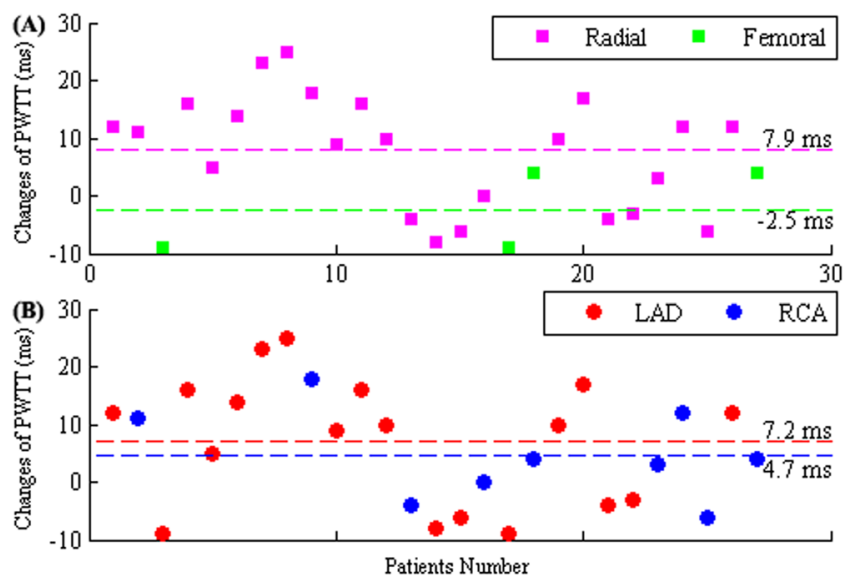

Figure 1. Scatter plots of the changes in PWTT in radial access group and femoral access group (A), as well as in LAD and RCA groups (B). Pink squares represent cases of patients in radial access group and green squares denote cases in femoral access group in section (A). In section (B), the red plots represent values of patients in LAD and the blue plots denote values in RCA. Mean changes of all four groups are shown by the dotted lines with corresponding color.

(A)

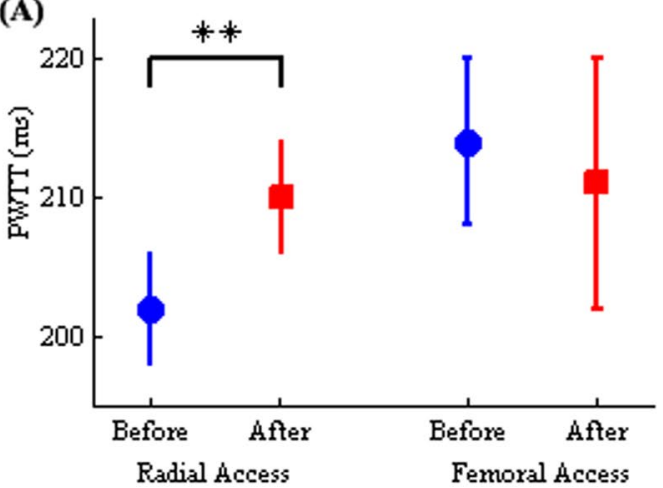

(B)

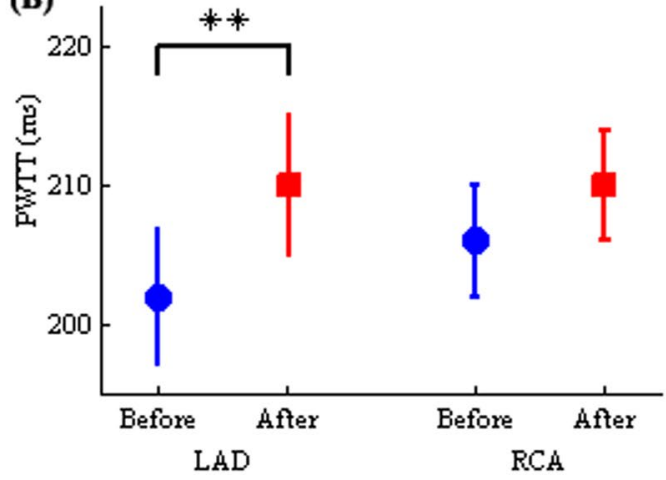

Figure 2. Effects of access sites and stent sites on PWTT in CAD patients. Influence of PCI from radial and femoral access sites (A), as well as with stent sites in LAD and RCA (B) on PWTT interval for CAD patients. $* *$ denotes $p<0.01$.

there was not a significant difference between the two procedures, there was only a slight increase in the PWTT (before $206 \pm 4 \mathrm{~ms}$ vs. after $210 \pm 4 \mathrm{~ms}, p>0.1$ ) for the RCA group.

\section{Discussion}

This study investigated the changes in PWTT within $24 \mathrm{~h}$ after PCI in CAD patients, and a significant increase in PWTT was observed. We also explored the effect of access and stent sites on the changes in PWTT. As a result, in the case of radial access and the LAD stent site, a significant increase in PWTT after PCI was observed. On the other hand, when the femoral access was chosen or the RCA stent implantation was performed, there were no significant changes in PWTT after PCI.

Kalay et al. ${ }^{9}$ have shown that carotid-femoral aortic PWV after PCI was significantly lower than before the procedure, which has been associated with a decrease in artery stiffness levels. Furthermore, Lang et al. and Tavil et $a l .^{20,21}$ have found that the decrease in artery stiffness is closely correlated with the enhancement of left ventricular functions. This could be explained because improved left ventricular function can increase the cardiac output, inhibiting the activity of the sympathetic nerve, and reducing tension of the artery while improving its elasticity. In this study, we found a significantly increased peripheral PWTT shortly after PCI, which indicates that PCI is helpful for improving arterial elasticity and the left ventricular functions. Other studies have shown that successful PCI should be able to enhance the left ventricular functions shortly after the procedure ${ }^{20,22}$. Thus, the increase of PWTT after the procedure can also be considered as an indicator of a successful PCI, and is measured by a noninvasive method. In addition, it has been reported that there was no significant difference in ambulatory blood pressure between 3-6 days after PCI and 1-3 days before PCI ${ }^{23}$. Our study also found that SBP did not significantly decrease within $24 \mathrm{~h}$ after PCI. The autonomic regulatory function and regulation mechanisms that perform blood pressure control functions have not yet been restored in the short period after PCI (e.g., 24h or 3-6 days); therefore, the balance in blood pressure cannot be recovered and retained to its preoperative value by this time. 


\begin{tabular}{|l|c|c|}
\hline Variables & Value & Range \\
\hline Number $(\mathrm{M} / \mathrm{F})$ & $27(15 / 12)$ & - \\
\hline Age $($ year $)$ & $62 \pm 9$ & $44-80$ \\
\hline Height $(\mathrm{cm})$ & $168 \pm 8$ & $150-181$ \\
\hline Weight $(\mathrm{kg})$ & $72 \pm 12$ & $42-91$ \\
\hline BMI $\left(\mathrm{kg} / \mathrm{m}^{2}\right)$ & $25.3 \pm 2.9$ & $20.8-28.7$ \\
\hline SBP $(\mathrm{mmHg})$ & $129 \pm 9$ & $114-150$ \\
\hline DBP $(\mathrm{mmHg})$ & $79 \pm 8$ & $63-93$ \\
\hline HR (beat $/ \mathrm{min})$ & $63 \pm 7$ & $50-77$ \\
\hline
\end{tabular}

Table 2. Basic characteristics of all enrolled CAD patients. Note: value is expressed as number (male/female) or mean \pm standard deviation (SD). BMI: body mass index, SBP: systolic blood pressure, DBP: diastolic blood pressure, HR: heart rate.

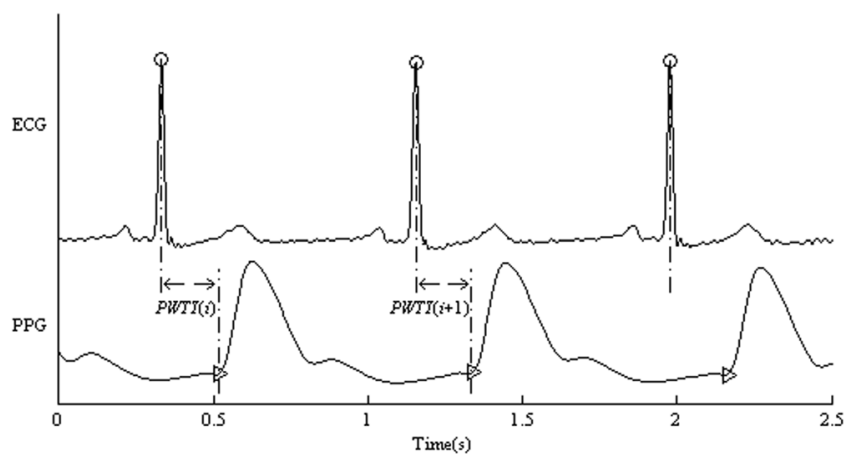

Figure 3. Construction process of PWTT time series from a segment of synchronously recorded ECG and PPG signals. " $O$ " denotes the R-wave peak and "D" denotes the foot of PPG. PWTT is defined as the interval from the R-wave peak to the foot of PPG signal in the same heart cycle.
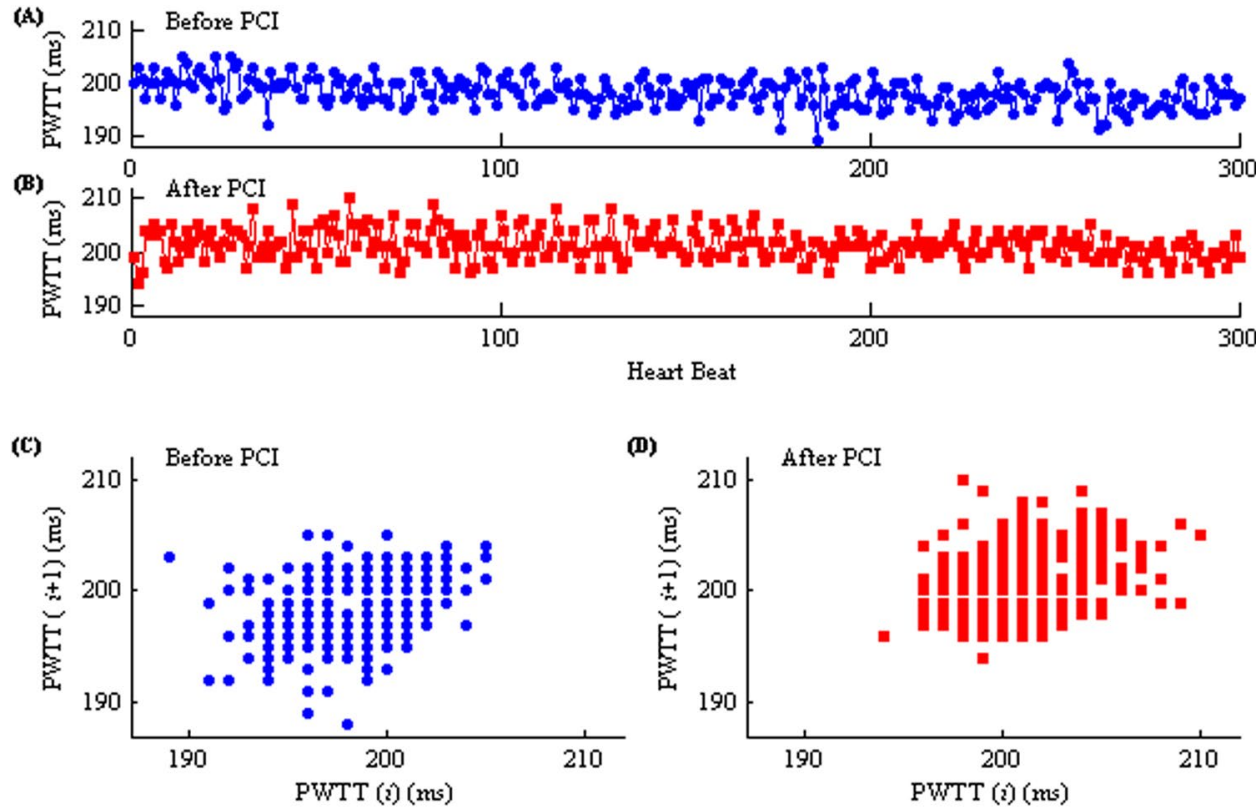

Figure 4. Examples of the PWTT interval series before (A) and after (B) PCI from one patient. Section (C) and (D) show the corresponding scatter plots of the two PWTT series.

It has been reported that compared with femoral access during $\mathrm{PCI}^{13,24,25}$, radial access has a stronger association with reduced adverse cardiovascular events. Guidelines from Europe ${ }^{26}$ have also endorsed the preferential use of radial access. In this study, PWTT significantly increased in the radial access group after PCI, whereas it 

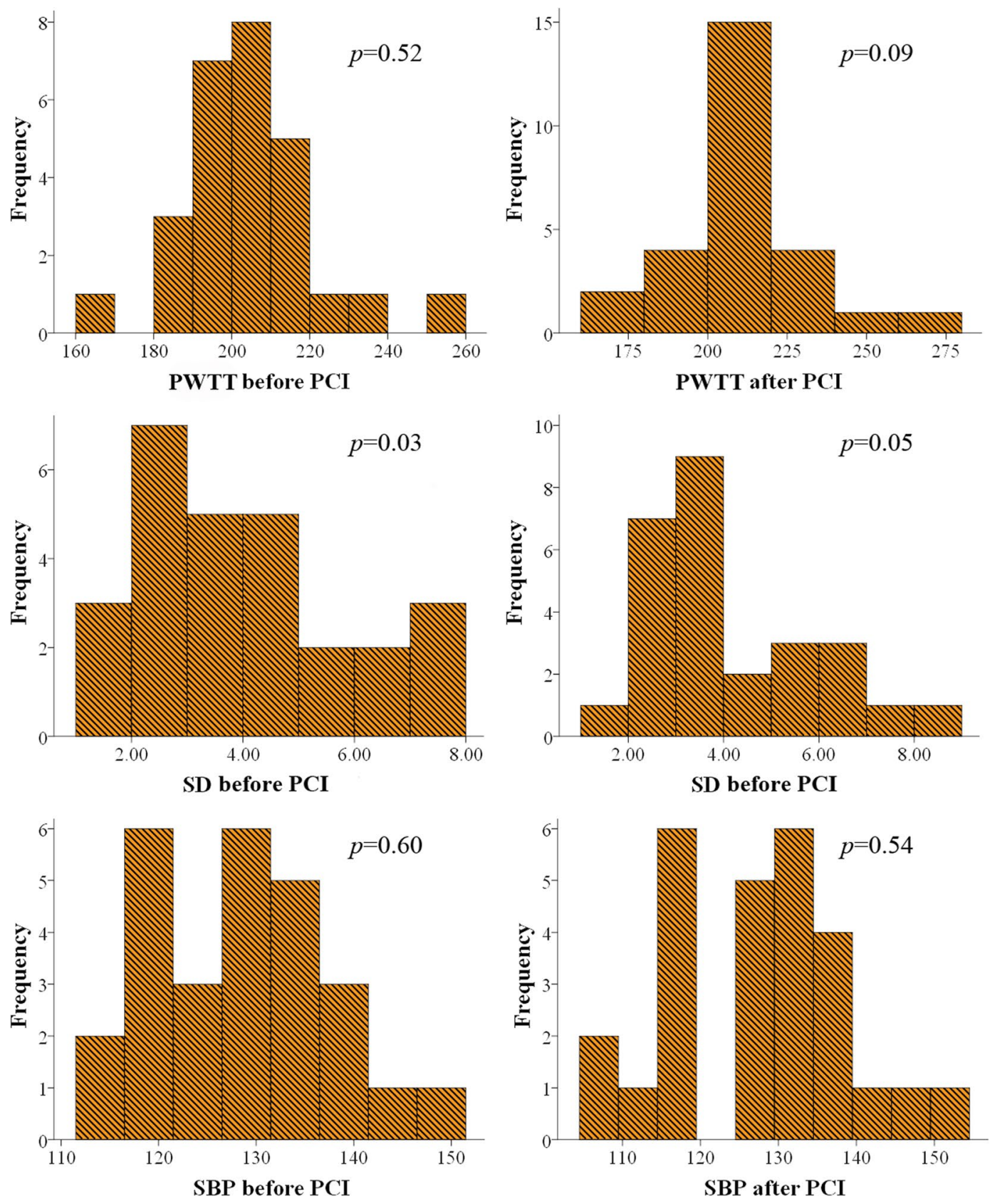

Figure 5. Results of normal distribution test of PWTT, SD and SBP before and after PCI.

displayed a decreasing trend in the femoral access group. These results indicate that radial access during PCI generated better outcomes than femoral access, which is in accordance with previous studies.

O'Keefe et al. ${ }^{16}$ and Holmes et al. ${ }^{17}$ suggested that patients with isolated LAD disease generally had positive revascularization results such as low in-hospital adverse event rates and good long-term outcomes. Our results show that patients with isolated LAD stents had significantly increased PWTT, while patients in the RCA group did not see an increase, which suggests that, within the short term after PCI, the artery status and left ventricular function of patients in the LAD group were improved compared to those in the RCA group. This might be attributed to the fact that the LAD supplies a large amount of the myocardium and is in close proximity to the distal left main coronary artery ${ }^{17}$, thus, revascularization here would contribute to the majority of the myocardium blood supply and improve the cardiovascular function. Additionally, Cortigiani et al. ${ }^{27}$ found that abnormal coronary flow reserve in the LAD had a higher annual hard event rate than abnormal coronary flow reserve in the RCA, which partially supports our results. Nevertheless, Kalay et al. ${ }^{9}$ reported that stents, both in the LAD and the RCA, brought about a significant decrease in PWV, which is not entirely consistent with our results. This might be due to our different measuring sites; the status of the aortas might be preferentially improved compared with the peripheral artery due to their closer proximity to the coronary artery. 
This study investigated the influence of PCI on the PWTT. Our results showed a significantly increased PWTT after the procedure, which indicated improved cardiovascular function and a successful PCI procedure. In addition, the influence of different stent and access sites on the PWTT was explored. The results suggested that radial access had better outcomes than femoral access, and revascularization in the LAD performed better than in the RCA. Nevertheless, the sample size of this study was relatively small, especially in the femoral access group and RCA group, and a further large-scale study is needed.

\section{Methods}

Subjects. Twenty-seven patients with coronary artery stenosis (aged between 44 and 80 ) were enrolled in this study. Routine ECG and echocardiography examinations were performed. Subjects who had frequent ectopic beats and left ventricular ejection fraction less than $50 \%$ were eliminated before participation. Patients were confirmed by coronary angiography, and all had at least one main coronary branch stenosed for over $50 \%$. Coronary angioplasty was performed according to established PCI standards. This clinical trial follows the declaration of Helsinki and regulations of China. CAD patients only were selected for this study; we did not include patients with other diseases such as diabetes mellitus, heart failure, etc. The study obtained full approval from the Institutional Review Board of Shandong Provincial Qianfoshan Hospital, and informed consent was required for each patient before participation. Basic characteristics of the patients are shown in Table 2.

Protocol. Measurements were taken in a quiet and temperature controlled room $\left(25 \pm 3^{\circ} \mathrm{C}\right)$ at Shandong Provincial Qianfoshan Hospital, Shandong University, using a cardiovascular function detection device (CV FD-II, inspection report number: D2012040606), which was produced by Huiyironggong Tech. Co., Ltd. (Jinan, China). Before commencing signal recording, each patient lay supine on a measurement bed for 10 minutes rest to allow for cardiovascular system stabilization. ECG electrodes were attached to the right wrist and to the right and left ankles to acquire a standard limb lead-II ECG. A photoelectric sensor was attached to the tip of the left forefinger to acquire finger PPG waves. The ECG and PPG signals were synchronously recorded at a sampling frequency of $1 \mathrm{kHz}$ for $5 \mathrm{~min}$ before and after the PCI procedure. Patients were told to breathe regularly and gently during the measurements. Blood pressure measurement was carried out with an arm blood pressure monitor after signal acquisition. The measurement was performed within $24 \mathrm{~h}$ before and after PCI by the same operator.

PWTT series construction. Slow varying components $(0-0.05 \mathrm{~Hz})$ in ECG and PPG signals were initially removed. ECG R-wave peaks were detected by a template-matching procedure ${ }^{28}$. With the location of R-wave peaks, the corresponding systolic feet were detected from the first-order differential signal of PPG ${ }^{29}$. PWTT series were constructed using the means of all intervals from the R-wave peaks to the feet of the corresponding PPG pulses in the same heart cycle ${ }^{3,30}$. Figure 3 demonstrates the construction process of PWTT time series from the synchronously recorded ECG and PPG signals. Anomalous intervals due to ectopic beats or poor signal quality were visually identified and removed from the PWTT time series.

Figure 4 shows examples of the PWTT series from before (A) and after (B) PCI procedures, as well as the corresponding scatter plots $(\mathrm{C}$ and $\mathrm{D})$ for the above-mentioned two PWTT series, respectively. The scatter plots in Fig. 4 section (D) are closer to the upper right than in section (C), which indicates that the values of beat-to-beat PWTT after PCI are more concentrated and higher than before PCI.

Statistical analysis. The individual mean PWTT value (PWTT) and standard deviation (SD) were calculated separately from the PWTT time series before and after PCI. The above calculations did not follow normal distributions (all $p<0.05 / 6$ using the Bonferroni correction), according to the results of the Shapiro-Wilk test which are shown in Fig. 5. Therefore, the Wilcoxon non-parametric test was used to verify significant differences of the PWTT and SD results, as well as SBP between two measurements, before and after the PCI procedure. Furthermore, patients were divided into two groups according to the stent site (i.e., LAD group and RCA group), who were also categorized in terms of the access site (i.e., radial access group and femoral access group). The significant differences between the two groups in each type were analyzed to determine the effects of stent site and access site on the changes of PWTT. Statistical analyses were performed using the SPSS software (Ver. 20, IBM, USA). Statistical significance was accepted at $p<0.05$.

Data availability. All data generated or analyzed during this study are included in this published article.

\section{References}

1. Smith, R. P., Argod, J., Pepin, J. L. \& Levy, P. A. Pulse transit time: an appraisal of potential clinical applications. Thorax. 54, 452-457 (1999).

2. Naschitz, J. E. et al. Pulse transit time by R-wave-gated infrared photoplethysmography: review of the literature and personal experience. Journal of clinical monitoring and computing. 18, 333-342 (2004).

3. Drinnan, M. J., Allen, J. \& Murray, A. Relation between heart rate and pulse transit time during paced respiration. Physiological measurement. 22, 425-432 (2001).

4. Zhang, Y. L., Zheng, Y. Y., Ma, Z. C. \& Sun, Y. N. Radial pulse transit time is an index of arterial stiffness. Hypertension research: official journal of the Japanese Society of Hypertension. 34, 884-887 (2011).

5. Kounalakis, S. N. \& Geladas, N. D. The role of pulse transit time as an index of arterial stiffness during exercise. Cardiovascular engineering (Dordrecht, Netherlands). 9, 92-97 (2009).

6. Mitchell, G. F. et al. Changes in arterial stiffness and wave reflection with advancing age in healthy men and women: the Framingham Heart Study. Hypertension. 43, 1239-1245 (2004).

7. Tedesco, M. A. et al. Effects of coexisting hypertension and type II diabetes mellitus on arterial stiffness. J Hum Hypertens. 18, 469-473 (2004) 
8. Bundhun, P. K., Soogund, M. Z. \& Huang, W. Q. Same Day Discharge versus Overnight Stay in the Hospital following Percutaneous Coronary Intervention in Patients with Stable Coronary Artery Disease: A Systematic Review and Meta-Analysis of Randomized Controlled Trials. PLoS One. 12, e0169807 (2017).

9. Kalay, N. et al. Effect of Significant Coronary Stenosis and Percutaneous Coronary Interventions on Aortic Stiffness. Turk Kardiyol Dern Ars. 40, 143-147 (2012).

10. Applegate, R. J. Radial access for primary percutaneous coronary intervention for ST-segment elevation myocardial infarction: time for a paradigm shift? J Am Coll Cardiol. 63, 973-975 (2014).

11. Arzamendi, D. et al. Effect on bleeding, time to revascularization, and one-year clinical outcomes of the radial approach during primary percutaneous coronary intervention in patients with ST-segment elevation myocardial infarction. Am J Cardiol. 106, 148-154 (2010).

12. Hetherington, S. L. et al. Primary percutaneous coronary intervention for acute ST-segment elevation myocardial infarction: changing patterns of vascular access, radial versus femoral artery. Heart. 95, 1612-1618 (2009).

13. Bertrand, O. F. et al. Comparison of transradial and femoral approaches for percutaneous coronary interventions: a systematic review and hierarchical Bayesian meta-analysis. Am Heart J. 163, 632-648 (2012).

14. Hannan, E. L. et al. Utilization of radial artery access for percutaneous coronary intervention for ST-segment elevation myocardial infarction in New York. JACC. Cardiovascular interventions. 7, 276-283 (2014).

15. Harris, P. J. et al. The prognostic significance of $50 \%$ coronary stenosis in medically treated patients with coronary artery disease. Circulation. 62, 240-248 (1980).

16. O’Keefe, J. H. Jr. et al. Isolated left anterior descending coronary artery disease: percutaneous transluminal coronary angioplasty versus stenting versus left internal mammary artery bypass grafting. Circulation. 100, II114-118 (1999).

17. Holmes, D. R. Jr. \& Bell, M. R. Left anterior descending artery stenosis: the widow maker revisited. Mayo Clin Proc. 75, 1113-1115 (2000).

18. Mahmarian, J. J., Pratt, C. M., Boyce, T. M. \& Verani, M. S. The variable extent of jeopardized myocardium in patients with single vessel coronary artery disease: quantification by thallium-201 single photon emission computed tomography. J Am Coll Cardiol. 17, 355-362 (1991).

19. O’Keefe, J. H. Jr. et al. Factors influencing myocardial salvage with primary angioplasty. Journal of nuclear cardiology: official publication of the American Society of Nuclear Cardiology. 2, 35-41 (1995).

20. Lang, T. W. et al. Consequences of reperfusion after coronary occlusion. Effects on hemodynamic and regional myocardial metabolic function. Am J Cardiol. 33, 69-81 (1974).

21. Tavil, Y. et al. The relationship between aortic stiffness and cardiac function in patients with obstructive sleep apnea, independently from systemic hypertension. J Am Soc Echocardiogr. 20, 366-372 (2007).

22. Remmelink, M. et al. Acute Left Ventricular Dynamic Effects of Primary Percutaneous Coronary Intervention: From Occlusion to Reperfusion. J. Am. Coll. Cardiol. 53, 1498-1502 (2009).

23. Yang, J., Yang, X., Liu, W. \& Cao, T. Effects of percutaneous coronary intervention on the ambulatory blood pressure of patients with hypertension and coronary heart disease. Irish journal of medical science. 184, 845-850 (2015).

24. Valgimigli, M. et al. Radial versus femoral access in patients with acute coronary syndromes undergoing invasive management: a randomised multicentre trial. Lancet. 385, 2465-2476 (2015).

25. Romagnoli, E. et al. Radial Versus Femoral Randomized Investigation in ST-Segment Elevation Acute Coronary Syndrome: The RIFLE-STEACS (Radial Versus Femoral Randomized Investigation in ST-Elevation Acute Coronary Syndrome) Study. J. Am. Coll. Cardiol. 60, 2481-2489 (2012).

26. Steg, P. G. et al. ESC Guidelines for the management of acute myocardial infarction in patients presenting with ST-segment elevation. Eur Heart J. 33, 2569-2619 (2012).

27. Cortigiani, L. et al. Prognostic correlates of combined coronary flow reserve assessment on left anterior descending and right coronary artery in patients with negative stress echocardiography by wall motion criteria. Heart. 95, 1423-1428 (2009).

28. Li, P. et al. A low-complexity data-adaptive approach for premature ventricular contraction recognition. Signal Image Video Process. 8, 111-120 (2014).

29. Yambe, T. et al. Clinical research on the accuracy in determining the pulse wave rising point. Scr. Med. 82, 164-174 (2009).

30. Ji, L. Z., Li, P., Li, K., Wang, X. P. \& Liu, C. C. Analysis of short-term heart rate and diastolic period variability using a refined fuzzy entropy method. Biomed. Eng. Online. 14, 64 (2015).

\section{Acknowledgements}

This study was supported by the National Natural Science Foundation of China (No. 61471223, 61671275), Science and Technology Project of Shandong Province (No. 2014GSF118030, 2015GSF118179), Natural Science Foundation of Shandong Province (No. ZR2015FQ016) and the Excellent Young Scientist Awarded Foundation of Shandong Province (No. BS2012DX019). We would like to thank all the patients and research fellows who participated in this study.

\section{Author Contributions}

L.J. and C.Y.L. analyzed the data; L.J., C.Y.L. and P.L. interpreted the results; L.J., C.Y.L. and P.L. wrote the manuscript; X.W. and Y.H. performed the experiments; C.C.L. designed and guided the study. All authors read and approved the final manuscript.

\section{Additional Information}

Competing Interests: The authors declare that they have no competing interests.

Publisher's note: Springer Nature remains neutral with regard to jurisdictional claims in published maps and institutional affiliations.

Open Access This article is licensed under a Creative Commons Attribution 4.0 International License, which permits use, sharing, adaptation, distribution and reproduction in any medium or format, as long as you give appropriate credit to the original author(s) and the source, provide a link to the Creative Commons license, and indicate if changes were made. The images or other third party material in this article are included in the article's Creative Commons license, unless indicated otherwise in a credit line to the material. If material is not included in the article's Creative Commons license and your intended use is not permitted by statutory regulation or exceeds the permitted use, you will need to obtain permission directly from the copyright holder. To view a copy of this license, visit http://creativecommons.org/licenses/by/4.0/.

(c) The Author(s) 2017 